\title{
LA ENSEÑANZA DE LA MEDICINA CLINICA EN LA VALENCIA DEL SIGLO XIX: LA LABOR DE JUAN BAUTISTA PESET Y VIDAL*
}

\section{Mariano Peset Mancebo}

\section{Introducción}

Durante el sexenio comprendido entre la revolución de 1868 y la restauración de la dinastía borbónica, la llamada "medicina de laboratorio» comenzó a desplazar en España a la anatomoclínica que había sido la dominante en la patología y la clínica del período isabelino. Este proceso de introducción ha sido estudiado principalmente desde el punto de vista de las instituciones y de las publicaciones dedicadas a las diversas áreas de la medicina (1). El presente trabajo tiene como objetivo contribuir al conocimiento de dicho período de nuestra historia médica, analizando una fuente que permite acercarse a la enseñanza clínica que se impartía

\footnotetext{
* Este artículo se ha realizado disfrutando el autor una beca pre-doctoral de la Consellería de Cultura, Educación y Ciencia de la Generalitat de Valencia. 
en el mismo. Se trata de dos volúmenes que recogen historias clínicas realizadas por los estudiantes de la Facultad de Medicina de Valencia durante los cursos 1872-73 y 1873-74, bajo la dirección de Juan Bautista Peset y Vidal. La destacada posición de esta figura en la medicina española de la época y la postura que había mantenido hasta entonces, confieren a la fuente en cuestión un particular interés, principalmente porque Peset y Vidal puede considerarse como la persona médica española del período isabelino que más se había significado como crítico de los planteamientos de la «medicina de laboratorio» desde una versión de la mentalidad anatomoclínica cercana a la "clínica pura» de Trousseau.

Recordemos que Juan Bautista Peset y Vidal, nacido en Gandía el año 1821, era hijo de Mariano Peset de la Raga, médico primario del Hospital General de Valencia, y sobresaliente figura, asimismo, de la medicina valenciana de la generación anterior. Tras estudiar medicina en la Facultad de Valencia desde 1834 a 1840, ejerció la profesión en las localidades de Motilla y Alcántara. En 1841 obtuvo una de las plazas de número del Hospital General, siendo también director facultativo del departamento de enajenados. El 14 de septiembre de 1854, fue nombrado facultativo de entradas del Hospital General. También desempeñó funciones en la universidad, siendo profesor de clínica médica y de medicina legal y toxicología durante la década de los setenta.

Durante la invasión de cólera de 1854, fue nombrado individuo de la comisión permanente de salubridad pública, y de la Junta Municipial de Sanidad. En esta epidemia, así como en la del 1855 y 1865, fue médico director de los hospitales de coléricos. Fue nombrado, también, médico de tercera de beneficencia, en 1862, y médico de segunda de beneficencia, con destino al departamento de enajenados. En 1866, fue vocal de la Junta Auxiliar de Cárceles. Dos años después, lo nombraron visitador del Hospital General. Durante la década de 1870 y 1880, fue médico vocal de la Junta Provincial de Sanidad, donde desempeñó la función de presidente en varias comisiones. Perteneció, también, a la comisión epidemiológica de la misma. El 22 de junio de 1881, fue cesado como vocal de la Junta Municipal de Sanidad.

Sin embargo, la mayor parte de su labor como médico la desarrolló en el Instituto Médico Valenciano. Desempeñó la función de secretario y perteneció a diferentes comisiones que tenía el Instituto. Durante el bienio 1864-1865, fue vicepresidente, siendo reelegido con posterioridad para los dos bienios siguientes. Además, fue elegido presidente para los años 18691870. 
Murió el 27 de junio de 1885, a los 64 años de edad, durante la epidemia de cólera que asoló la ciudad de Valencia en ese año(2).

\section{Las historias clínicas y su comentario estadístico por Peset y Vidal}

Los dos volúmenes antes citados, incluyen un total de sesenta y dos historias clínicas realizadas por los alumnos que dirigía Peset y Vidal. Corresponden a los años 1872-73 y 1873-74. Llevan ambos el título de Historias clínicas a cargo del Dr. D. Juan Bautista Peset y están editados por la imprenta valenciana de Ferrer de Orga, en 1873 y 1874.

En el volumen del curso 1872-73 aparece un prólogo de la comisión de estudiantes que redactaron las historias y una introducción estadística del propio Peset y Vidal de la que trataremos más adelante. De los cinco miembros que firman el citado prólogo, dos de ellos fueron después notables figuras médicas: Luis Simarro Lacabra y Enrique Salcedo Ginestal. En el prólogo se exponen los "propósitos» del libro: reunir en un pequeño volumen los casos más representativos del curso de clínica, imprimiendo la colección para ahorrar tiempo. Se evitan así los errores de la copia manuscrita, consiguiendo "por este medio una autenticidad de datos y uniformidad de conocimientos imposible con el sistema antiguo, que abandonaba a la actividad individual el trabajo coleccionador y ofrecer un conjunto concreto y conciso de las historias sobre las que han de versar los exámenes de fin de curso»(3). Lo consideran un recuerdo de compañerismo, así como un retrato intelectual de los autores en el momento más importante y decisivo de la vida académica. Por último, advierten que suprimen los diagnósticos diferenciales, que se reducen a simples indicaciones. Indican que no se han publicado las 107 historias clínicas que figuran en la estadística por la brevedad, economía y carácter compendiado del libro. En cambio, recogen todas ellas en una estadística que sirva de base a estudios generales.

Esta recopilación termina con las tablas estadísticas sobre las que el propio Juan Bautista Peset y Vidal ofrece una breve introducción. Comienza diciendo que «el criterio de cantidad que surge de las aplicaciones de la estadística se presenta al espíritu con certidumbre matemática, y no es, por tanto, extraño que se le haya atribuido un valor y una importancia capitales en todas las ciencias...»(4), sobre todo en medicina, en donde se refiere al estudio y observación de numerosos casos clínicos unidos entre sí por relación de semejanza o disparidad que permiten clasificarlos. De todas formas - sigue diciendo- muchas veces el problema es insoluble, 
pudiendo decirse que más bien es una forma más exacta de plantearlas que de solucionarlas.

Estas consideraciones no tienden a desacreditar el método estadístico e invalidar sus resultados, sino a subrayar el carácter auxiliar de la experiencia. En un momento se creyó que iba a resolver todos los problemas, mas todo ello no fue sino una forma de llamar la atención sobre ideas nuevas de manera que transforman la «utopía quimérica y estéril en principio práctico y fecundo» (5).

Los números por sí solos - según Peset y Vidal- expresan relaciones. de cantidad, pero combinados revelan leyes íntimas de los fenómenos más complicados. Sin embargo, el método númerico ofrece oráculos ambiguos o soluciones ajustadas al deseo del investigador. Esta ambigüedad - dice Peset- depende tal vez de la dificultad de expresar con guarismos la complicación propia de los fenómenos patológicos y terapéuticos. Estos hechos constituyen los defectos centrales de la estadística y multiplican las causas de error, así como el exceso numérico de hechos, el carácter y condiciones especiales de los recogidos y la falta de detalles respecto de los mismos.

La interpretación estadística, que es la inducción misma, exige, como ésta, gran prudencia para eludir las causas de error y una sutil sagacidad para descubrir el significado de cada cifra. Hay fenómenos - sigue diciendo- que ofrecen algunas circunstancias cuyo descubrimiento es absolutamente independiente de la cantidad de hechos observados; en otros los números son jueces en donde la solución sólo está en la estadística numerosa y completa. En muchos, las cifras descubren coincidencias que inducen al descubrimiento de nuevos principios, como la herencia patológica.

El estudio de las cifras es lo más importante de la estadística, «es el que mayormente necesita de severa escrupulosidad en el análisis de los datos y de inflexible lógica en su inducción, evitando así el considerar como generales los resultados que sólo tienen una significación particular y erigir en absoluto lo que depende de condiciones más o menos determinadas» (6). Existen algunas causas de errores, como son la pobreza de los individuos, la cronicidad de las afecciones, las causas debilitantes que afectan a los enfermos, la falta de cuidados al principio de la enfermedad y la gravedad ordinaria de ésta, como también las malas condiciones higiénicas, el hacinamiento de los enfermos y las epidemias que son su consecuencia. Todo esto hace que los registros de los hospitales tengan un tono sombrío que «desacreditaría la medicina al no tener presente que estas circunstancias revisten a la estadística hospitalaria del carácter del 
complemento de la estadística civil, donde condiciones opuestas forman la parte clara del cuadro de la medicina práctica»(7).

Por último las estadísticas de clínica, según Peset y Vidal, conducirán a las mayores aberraciones si, olvidando su carácter especial, se pretendiera deducir de ellas leyes generales. Asi, en estas estadísticas, el número de hombres duplica al de las mujeres, pero de aquí no podrá deducirse en modo alguno la mayor frecuencia de enfermedades entre los varones sino el mayor pudor y vergüenza propios de la mujer que le hacen acudir menos a las consultas.

El resto de la introducción lo dedica a comentar las tablas.

Reproduciremos a continuación las tablas de los ciento siete enfermos asistidos añadiendo, al lado, los datos de los treinta y cuatro seleccionados. Corresponden a los siguientes apartados: temperamento, estado civil, edades, profesiones, diagnósticos, altas y autopsias.

TABLA I

TEMPERAMENTOS

\begin{tabular}{|c|c|c|c|}
\hline Seleccionados & Asistidos & Seleccionados & $\%$ \\
\hline Linfáticos & 41 & 8 & 19,51 \\
\hline Sanguíneos & 24 & 12 & 50 \\
\hline Sanguíneos-Linfáticos & 14 & 2 & 14,28 \\
\hline Nerviosos & 13 & 2 & 15,38 \\
\hline Linfo-Nervioso & 6 & 4 & 66,66 \\
\hline Sanguíneo-Nervioso & 9 & 1 & 11,11 \\
\hline Sanguíneo-Bilioso & - & 2 & - \\
\hline NO CONSTA & - & 3 & - \\
\hline TOTAL & 107 & 34 & \\
\hline
\end{tabular}


TABLA II

ESTADO CIVIL

\begin{tabular}{|c|c|c|c|}
\hline & Asistidos & Seleccionados & \% Seleccionados \\
\hline Solteros & 34 & 13 & 38,23 \\
\hline Casados & 57 & 13 & 22,80 \\
\hline Viudos & 16 & 6 & 37,5 \\
\hline NO CONSTA & - & 2 & - \\
\hline TOTAL & 107 & 34 & \\
\hline
\end{tabular}

Peset y Vidal considera por separado los hombres y las mujeres, encontrando que hay un predominio de hombres casados. Esto lo interpreta aduciendo que existe malestar y miseria en los matrimonios pobres, lo que hace que la enfermedad del marido sea más penosa que la de la mujer, ya que se «ciega por completo las fuentes de subsistencia y hace forzoso recurrir al Hospital» (8).

\section{TABLA III}

\section{EDADES}

\begin{tabular}{|c|c|c|c|}
\hline & Asistidos & Seleccionados & $\%$ Seleccionados \\
\hline Menor de 10 & 1 & 0 & 00,00 \\
\hline Menor de 21 & 22 & 6 & 27,27 \\
\hline $21-40$ años & 51 & 20 & 37,25 \\
\hline Mayor de 40 & 33 & 8 & 24,24 \\
\hline TOTAL & 107 & 34 & \\
\hline
\end{tabular}


TABLA IV

OFICIOS EJERCIDOS AL AIRE LIBRE O EN EL CAMPO

\begin{tabular}{|c|c|c|c|}
\hline & Asistidos & Seleccionados & $\%$ Seleccionados \\
\hline Labradores & 21. & 6 & 23,80 \\
\hline Jornaleros & 5 & 2 & 40 \\
\hline Carabineros & 4 & 3 & 75 \\
\hline Marineros & 3 & - & - \\
\hline TOTAL & 33 & 11 & \\
\hline
\end{tabular}

TABLA V

OFICIOS EJERCIDOS EN LAS GRANDES POBLACIONES

\begin{tabular}{|c|c|c|c|}
\hline & Asistidos & Seleccionados & \% Seleccionados \\
\hline Albañiles & 7 & 2 & 28,57 \\
\hline Zapateros & 3 & 3 & 100,00 \\
\hline Arte de la seda & 3 & - & - \\
\hline Cocheros & 3 & - & - \\
\hline Carpinteros & 2 & 1 & 50,00 \\
\hline $\begin{array}{l}\text { Guanteros, litógrafos, } \\
\text { sogueros, pintores, } \\
\text { torneros, etc. (uno de } \\
\text { cada oficio }\end{array}$ & 19 & 6 & 31,57 \\
\hline TOTAL & 37 & 12 & \\
\hline
\end{tabular}




\section{TABLA VI}

PROFESIONES DE LAS MUJERES

\begin{tabular}{|c|c|c|c|}
\hline & Asistidos & Seleccionados & \% Selecciones \\
\hline Criadas de servicio & 11 & 3 & 27,27 \\
\hline Costureras & 6 & 5 & 83,33 \\
\hline \multicolumn{4}{|l|}{ Dedicadas al cuidado } \\
\hline de su casa & 5 & - & - \\
\hline Cigarreras & 3 & - & - \\
\hline Labradoras & 2 & - & - \\
\hline Nodrizas & 2 & - & - \\
\hline \multicolumn{4}{|l|}{$\begin{array}{l}\text { Lavanderas, Carnice- } \\
\text { ras, Calceteras, etc. }\end{array}$} \\
\hline (de cada oficio una) & 8 & 1 & 12,5 \\
\hline TOTAL & 37 & 9 & \\
\hline
\end{tabular}

TABLA VII

ESTADO DE LAS ALTAS

\begin{tabular}{|c|c|c|c|}
\hline & Asistidos & Seleccionados & $\%$ Seleccionados \\
\hline Curados & 53 & 10 & 18,86 \\
\hline Aliviados & 41 & 17 & 41,46 \\
\hline Muertos & 13 & 7 & 53,84 \\
\hline TOTAL & 107 & 34 & \\
\hline
\end{tabular}

\section{TABLA VIII}

\section{AUTOPSIAS}

\begin{tabular}{lccc}
\hline & Asistidos & Seleccionados & \% Seleccionados \\
\hline REALIZADAS & 13 & 7 & 53,84 \\
NO REALIZADAS & 94 & 27 & 28,72 \\
\hline
\end{tabular}


TABLA IX

ENFERMEDADES (*)

\begin{tabular}{lccc}
\hline & Asistidos & Seleccionados & \% Seleccionados \\
\hline Pulmonar & 12 & 11 & 91,66 \\
Digestivo & 16 & 6 & 37,5 \\
Corazón & 11 & 3 & 27,27 \\
Infeccioso & 11 & -3 & 54,54 \\
Nervioso & 10 & 2 & 30,00 \\
Ginecología & 3 & 3 & 66,66 \\
Locomotor & 9 & 33,33 \\
\hline
\end{tabular}

* Esta última tabla se ha realizado según las especialidades actuales.

En la tabla III vemos que no existe más que un individuo de menos de 10 años. Esta ausencia de niños en el hospital es debida, según Peset, a la agudeza de las enfermedades infantiles y, sobre todo, a que las madres más pobres tienden a separarse menos de sus hijos. Hay un predominio de enfermos con edades comprendidas entre los 21 y 40 años, lo que lleva a considerar que existe una mayor frecuencia de enfermedades crónicas.

De las tablas de profesiones se deduce que los hospitalizados proceden de las capas más bajas de la sociedad, predominando carabineros y jornaleros. En cuanto a los labradores, Peset y Vidal destaca que hay ocho casos de paludismo, enfermedad a la que sólo están expuestos los habitantes de los campos en donde se cultive el arroz. Los labradores no tienen asistencia médica gratuita, al contrario que los pobres de la ciudad, lo que contribuye a disminuir el número de estos en el hospital. Entre las mujeres, el oficio predominante entre las seleccionadas es el de costurera aunque haya un mayor número de criadas entre el total de las enfermas asistidas.

En las tablas VII y VIII hay que considerar como conclusión importante que, de las trece defunciones ocurridas entre los enfermos asistidos, se realizan autopsias en todas ellas, ocurriendo lo mismo entre los casos seleccionados. La autopsia era realizada en todos los casos de muerte como método diagnóstico seguro, de acuerdo con los principios de la mentalidad anatomoclínica de Peset y Vidal. Las autopsias fueron realizadas indiscriminadamente desde una turberculosis pulmonar hasta una hepatitis o una anemia. 
TABLA X

ENFERMEDADES DE LOS 107 ENFERMOS ASISTIDOS

\begin{tabular}{lrlr}
\hline Erisipela & 2 & Hipocondria & 1 \\
Viruela & 1 & Mielitis & 2 \\
Calentura tifoidea & 2 & Enteralgia & 1 \\
Intermitentes y paludismo & 14 & Anginas & 2 \\
Reumatismo & 11 & Gastritis y Enterocolitis & 7 \\
Clorosis & 1 & Tenia & 1 \\
Sífilis & 1 & Hernia & 1 \\
Infarto, bazo e hígado & 1 & Hepatitis y Ascitis & 3 \\
Diatesis calculosa & 2 & Ataxia locomotrix & 1 \\
Cáncer de rinón & 1 & Corea & 1 \\
Metrorragia, Vaginitis, Prolapso & 4 \\
de útero & & Neuralgia y gastralgia & 3 \\
Endocarditis & 3 & Bronquitis aguda & 1 \\
Aneurisma & 2 & Catarro pulmonar crónico & 1 \\
Congestión cerebral & 3 & Hidroneumotorax & 10 \\
Apoplegía & 1 & Turberculosis y tisis & 5 \\
Vértigo & 1 & Hipertrofia cardíaca & 4 \\
Demencia & 1 & Estrechez valvular & \\
\hline
\end{tabular}

En las dos últimas tablas referentes a las enfermedades podemos ver que las pulmonares son las predominantes entre los seleccionados, quizá por ser en éstas en donde las autopsias proporcionan más datos. En la última tabla vemos que las afecciones que predominan son las palúdicas, seguidas de las pulmonares, lo que corresponde a la importancia social que en esta época tenían las primeras.

En el segundo volumen de historias clínicas dirigidas por Peset y Vidal pertenecientes al curso de 1873 a 1874, encontramos un total de ocho autopsias. Las autopsias son también consideradas como parte fundamental en las historias clínicas, son importantes tanto para el diagnóstico como para el pronóstico de la enfermedad, ya que pueden ser distintos según se haya visto en ella una u otra lesión. El siguiente texto es claro ejemplo de esta actitud expectante del médico anatomoclínico: «La observación en las autopsias de lesiones gravísimas incompatibles con la vida, que no se hayan manifestado durante ésta por ningún género de síntomas, ocurre con bastante frecuencia en la práctica; cuyo hecho nos 
indica la necesidad de ser reservados y cautos en los diagnósticos y pronósticos, contribuyendo de esta manera a sostener la reputación del médico».(9)

Anotemos, por último, que una de las historias clínicas de este volumen fue redactada por Francisco Barberá Martí, figura importante dentro de la otorrinolaringología y de la historia de la medicina valencianas. La historia corresponde a un cáncer de estómago en donde se puede ver un interesante diagnóstico diferencial, entre el cáncer y la úlcera de estómago.

3. El contenido de las historias clinicas: el tránsito de la medicina anatomoclinica a la de "laboratorio»

Como sabemos, Peset y Vidal fue seguidor de la mentalidad anatomoclínica. Utilizaremos el análisis del método anatomoclínico expuesto por Laín Entralgo en su libro La Historia Clínica (1961), para encuadrar el contenido de las historias clínicas por él dirigidas.

Recordaremos únicamente que, según Laín Entralgo, la historia clínica, desde Boerhaave, tiene cinco apartados: descripción del sujeto, antecedentes - remotos y próximos-, estado presente, relato del proceso morboso y exitus con curación, curación con defecto o muerte con lo que se realizará la autopsia. A estos apartados habrá que añadir, por supuesto, el tratamiento, tradicional en la mayoría de las historias clínicas dirigidas por Peset y Vidal. En ellas encontramos tres apartados: "parte expositiva», que consta de "preámbulo», "antecedentes o conmemorativo», y «estado actual»; "parte razonada», integrada por el «diagnóstico», "pronóstico», "curso», "duración», "etiología» y "tratamiento»; "parte complementaria» con un «diario clínico», «autopsia» y "reflexiones».

El «Preámbulo» corresponde a lo que Laín llama descripción del sujeto, en donde se apunta el «nombre», «origen», «edad», «residencia», «temperamento», "constitución», "profesión», "sexo» del enfermo. Recordemos que el «temperamento» o «constitución» es una parte importante en las historias clínicas tradicionales, como propiedades hereditarias potenciales que el individuo lleva desde el nacimiento y se manifestarán o no dependiendo de las circunstancias del medio que le rodea, es decir, según el modo de reaccionar del individuo a las causas patógenas exteriores(10). Según López Piñero, la mención del temperamento en las historias clínicas de Peset «queda luego sin relación, ni peso de ninguna clase en el resto de los elementos del relato patográficon(11). En definitiva, la constitución es un concepto olvidado de aparición rutinaria. 
Los "antecedentes» son importantes desde el punto de vista etiológico, tanto los referentes a la familia del enfermo como los del propio paciente antes de llegar a la clínica. En algunas de las historias clínicas, realizadas bajo la dirección de Peset y Vidal, aparecen antecedentes familiares como en el caso de una «ataxia locomotriz» progresiva: «Su padre padeció siempre de dolores que le impedían andar con libertad. Murió hace seis meses de una afección de pecho. Su madre goza de inmejorable salud. Su abuelo materno murió tísico. Los hermanos de su padre han padecido igual padecimiento que ella; y de los tres hermanos de la enferma, el mayor de 14 años, sufre de igual suerte, temiendo la familia le suceda otro tanto a una niña de 9 años» (12). Los antecedentes del enfermo pueden ser remotos o próximos, los primeros hasta el comienzo de la enfermedad, los segundos, desde el comienzo hasta el primer contacto con el patólogo(13). Transcribiré como ejemplo los de un enfermo de «tuberculosis» pulmonar donde se puede distinguir tanto unos como otros. Padece - se lee- "de unas tercianas a los 13 años; una angina a los 18, y bañándose un día en Cartagena, a los 20 añós, observó que le sobrevino un golpe de tos acompañado de la salida de alguna cantidad de sangre. Esta hemoptisis se repitió después más tarde por espacio de siete veces consecutivas y a la segunda ya comenzaron los padecimientos del aparato respiratorio, iniciándose por una tos seca primero y acompañada luego de espectoración con ligero dolor de pecho"(14). Hasta aquí los antecedentes remotos del enfermo con el comienzo de la enfermedad. A estos padecimientos se añade en mayo de 1871 "un ligero dolor que empezando en la parte posterior, se extendía hasta el occipucio, llegando a veces hasta la frente... Así permaneció unos cuantos meses, hasta que un día de Diciembre, estando tomando el fresco a la puerta de su casa, notó al acostarse que el dolor se había aumentado de tal modo que le impidió dormir aquella noche». Por último, «viendo, pues, que su afección torácica iba en aumento, y que el dolor de la cabeza le incomodaba muchísimo, se decidió a venir a la clínica en la fecha antedicha»(15).

El «estado actual» incluye el estado presente y el relato del proceso morboso según el esquema de Laín Entralgo. El diagnóstico tiene un apartado especial que luego comentaremos. En este epígrafe aparecen ténicas de exploración como la palpación, la percusión y la auscultación típicas de la mentalidad anatomoclínica. Sin embargo, junto a ellas, figura también una técnica tan caracterizada de la mentalidad fisiopatológica como la termometría. Por último, hay que destacar la detección objetiva de parásitos como manifestación incipiente de la mentalidad etiopatológica. Veamos separadamente las técnicas utilizadas en estas historias. 
La palpación es una técnica exploratoria utilizada muy a menudo en nuestras historias clínicas. Por ejemplo, en una historia correspondiente a una "ascitis sintomática», se afirma: «Por la palpación, se notó fluctuación en la cavidad peritoneal, manifestándose bien clara la sensación de oleaje»(16) por el líquido acumulado en dicha cavidad. Es el denominado «signo de oleaje» utilizado actualmente para el diagnóstico de líquido en la cavidad peritoneal. En el relato patográfico aparece también la simple descripción externa del paciente, "cara triste demacrada, que indicaba largos padecimientos... piel de color amarillo terroso, seca y apergaminada; las regiones infradiafragmáticas aumentadas de volumen y con la piel distendible reluciente y algo transparente, notándose algunas redes venosas más dilatadas» (17), signo de hipertensión portal. Un caso de "pleuroneumonía» proporciona un ejemplo de palpación en la región torácica. «La palpación nos hizo distinguir que los movimientos del corazón eran más fuertes e impulsivos pero sin salir de los límites fisiológicos» (18).

El empleo de la percusión puede ejemplificarse en un caso de «encefaloides de riñón»: «En la parte inferior del vacío izquierdo se percibe por la palpación un tumor redondeado, sin abollonaduras, de 10 a 12 centímetros de diámetro, duro, indolente, inmóvil y que se desliza por debajo de las paredes abdominales. La percusión demostraba el sonido mate del tumor mismo"(19). También es utilizada en este mismo caso la exploración digital por los orificios naturales del cuerpo para determinar la posición exacta del tumor.

Como es sabido, la auscultación es una de las más importantes aportaciones semiológicas del método anatomoclínico. Esta técnica es utilizada rigurosa y constantemente en las historias clínicas dirigidas por Peset y Vidal. Así, en una «tisis pulmonar», se ausculta «una disminución muy pronunciada del murmullo vesicular, reemplazada por un soplo áspero con aumento de la vibración de las paredes torácicas, excepto en el vértice derecho, donde se oye con mucha claridad el estertor cavernoso y la pectoriloquia»(20). En otros casos, la exploración proporciona muchos más datos, como ocurre en una «estrechez e insuficiencia de las válvulas aórticas» donde, «Al auscultar, la cabeza del observador es rechazada por la fuerte impulsión cardíaca y se percibe el ruido del fuelle que principia después del primer ruido normal, llenando todo el segundo tiempo y gran parte del silencio. Este ruido de fuelle se modificó más adelante en el de lima o escofina y hasta en el de sierra. También es digno de atención que el ruido anormal se perciba con gran claridad en todo el trayecto de la aorta» (21). Sólo con estos datos - muy parecidos a los proporcionados en 
una historia actual—es posible el diagnóstico de una lesión de las válvulas sigmoideas y la estenosis de la misma.

El signo físico desde el punto de vista anatomoclínico, se refiere inmediatamente a una lesión local; si un dato no cumple esta exigencia no es, en rigor, físico. El fisiopatólogo no lo niega pero no admite su tajante exclusividad. Hay «signos físicos» que no «significan» lesiones anatómicas locales sino alteraciones del proceso químico y energético de la vida. Vaya o no acompañado de un desorden anatómico local, la fiebre constituye un transtorno morboso típico en el proceso evolutivo de la temperatura humana(22). El pulso y la disnea se pueden también considerar, desde este punto de vista, como síntomas. Todos estos son considerados como datos importantes a la hora de establecer un juicio diagnóstico en las historias clínicas dirigidas por Peset y Vidal. «En la circulación se notaba: pulso febril, pequeño y depresible (116 por minuto); recargos vespertinos, a los cuales seguían sudöres abundantes en la mitad superior del cuerpo y palmas de las manos, más generales y copiosos en la madrugada" (23). El termómetro, aunque de un modo menos sistemático que Wunderlich, es utilizado como método de investigación para saber el curso de una enfermedad en muchas de las historias clínicas: «su temperatura $39^{\circ}$ centígrados». El pulso y la respiración (disnea) son también síntomas tomados en consideración. «El pulso es frecuente y lleno (96 por minuto). En el aparato respiratorio encontramos palabra entrecortada, respiración acelerada y las ventanas de la nariz abiertas» (24). Son utilizados, o por lo menos conocidos, aparatos o técnicas claramente pertenecientes a la mentalidad fisiopatológica. Así, en un «reumatismo muscular» se usa el esfigmógrafo de Marey para la objetivación del pulso; "pues el ruido de roce que se aprecia por la auscultación en el segundo tiempo y el isocronismo que guarda este ruido con la pulsación irregular de la radial, así lo demuestran y así lo comprobaría quizá el esfigmógrafo de Marey.... (25). De manera incipiente podemos ver la influencia de la mentalidad fisiopatológica en estas historias clínicas, aunque ésta no puede ser considerada como una de las bases de la obra de Peset y Vidal. Sus sucesores en la Cátedra de Clínica de Valencia, Francisco Moliner y Julio Magraner, pueden, en cambio, considerarse adscritos a la citada mentalidad, ya desarrollada en todos sus aspectos.

El análisis de la orina como signo funcional es también utilizado en estas historias clínicas. Unas veces se describe a simple vista, «orinas sedimentosas y escasas». Otras se analiza químicamente -claro ejemplo de mentalidad anatomoclínica- como ocurre en una «insuficiencia valvular» en donde «las orinas eran escasas y de color rojo, en su presencia 
notábanse sedimentos precipitados y el análisis químico nos evidencia mucha albúmina, sin duda contendría otros elementos que el microscopio nos hubiese aclarado» (26). Hay historias clínicas en las que el análisis de orina se considera indispensable para el diagnóstico de la enfermedad. Así, en una "hidropesia ascitis sintomática», se afirma: "La sola inspección y análisis de la orina, que es donde se encuentran los síntomas patognomonicos de esta degeneración granulosa de parénquima renal, bastaron para persuadirnos que no existía en esta enfermedad tal alteración orgánica. En efecto; tratada la orina con el ácido nítrico primero, y con la ebullición más tarde, no pudimos comprobar en ella la presencia de la albúmina, sustancia que con cualquiera de estos procedimientos hubiésemos visto precipitarse rápidamente en el fondo de la vasija, en forma de coágulo, más o menos abundante y copioso, al ser una albuminuria la afección principal que esta enferma padecíar(27). Como se ve manifiestamente en este pasaje, no se buscaba una alteración funcional del riñón -es decir, no tenían en su mente conceptos fisiopatológicos- sino un signo que demostrara la alteración anatómica, en definitiva, la lesión renal. Esta desencadena la presencia de albúmina en las orinas, que será el síntoma patognomónico o signo que demuestra la lesión anatómica renal que en este caso es una nefritis albuminosa.

Para la mentalidad etiopatológica la enfermedad, como es sabido, es una alteración de la vida orgánica consecutiva a la infección de un germen, es una reacción vital, no una alteración cuantitativa del estado morboso o normal. El microbio o parásito - en nuestro caso- determina y configura la enfermedad. La patografía será un informe acerca de la inquisición del agente patógeno y un relato de las "consecuencias" por la penetración del agente en el organismo (28). En las historias clínicas que nos ocupan el caso de una Tenia Solium ejemplifica esta mentalidad en su etapa premicrobiológica, así como el uso del microscopio para la objetivación del agente causal. "Hace unos seis meses observó en sus excrementos, al tiempo de defecar, unos cuerpecillos blancos, lisos, dotados de un ligero movimiento, de 8 milímetros de ancho por 14 milímetros de largo... Se sospechó desde un principio que las semillas de melón que el enfermo acusaba no podrían ser más que las pequeñas articulaciones estrechas y largas de que está compuesta la tenia... En esta creencia estábamos cuando vino a corroborar el diagnóstico la circunstancia de arrojar el enfermo, el día 21 por la mañana, una pequeña tenia, cuya longitud era de 4 decímetros y una anchura de 7 milímetros; una de sus extremidades era abultada, observándose a simple vista unos pequeños tubérculos demostrándonos el microscopio que se trataba de la cabeza; la extremidad opuesta presen- 
taba el aspecto filiforme, el todo era aplanado y articulado, de color blanco amarillento, carácter que le distingue perfectamente del botriocéfalo que es de color grisáceon (29).

La bacteriología estaba en sus principios y en todas las historias de enfermedades infecciosas no se alude, por supuesto, al germen causal de la enfermedad. Se puede ver como en una historia clínica, se atribuye la causa del paludismo a la acción patógena de los campos de arroz, no llegando ni siquiera a plantear la existencia de un posible gérmen.

La utilización del microscopio es práctica iniciada por estas fechas -1873- en la Facultad de Medicina de Valencia. En la historia clínica de una "cirrosis hepática» encontramos la siguiente indagación: «El examen microscópico de una porción de hígado dio a conocer que esta víscera estaba afecta de cirrosis»(30). En esta época los médicos y cirujanos valencianos comenzaron a conceder a la histopatología una gran importancia, para aclarar problemas patológicos y clínicos. De esta forma, en 1873 se celebra un claustro en favor de la histología, como protesta de la única cátedra de esta materia creada en Madrid. En el punto cuarto del correspondiente acuerdo se dice: «Que en las clínicas, cuando algún caso patológico lo requiere para establecer el diagnóstico de una dolencia, de un modo fijo y preciso, o para comprobar el previamente formado, se recurre a las demostraciones de los elementos morbosos y de las metamorfosis que los tejidos experimentan, lo cual constituye conocimientos detallados en anatomía patológica»(31).

La «Parte Razonada» de la historia clínica incluye «Diagnóstico» «Pronóstico», «Curso», «Duración», «Etiología» y "Tratamiento» de la enfermedad. El diagnóstico se basa, en muchos casos, en los signos y síntomas recogidos en la exploración realizada en el apartado anterior (32). «La caracterización (o el diagnóstico) de cada especie morbosa se realiza por una detenida consideración de su sintomatología así como de los signos lesionales de auscultación; percusión, etc. Destacado lugar ocupa también el protocolo de autopsia. La concepción básica es, en suma, la anatomoclínica. En torno a la idea de lesión gira toda la nosología de Peset»(33).

El contenido de todas las autopsias es claramente anatomoclínico. Se busca en el cadáver "la lesión fija y constata la lesión específica»(34). para fundamentar la especie morbosa. En la autopsia de una «tisis pulmonar» se describe detalladamente la lesión cavernosa: «En el vértice del pulmón derecho una caverna doble de grandes dimensiones que comunicaba con los bronquios; en la base y parte posterior del mismo pulmón se puede observar otra caverna también de bastante estensión y en el pulmón izquierdo se vieron otras de menores dimensiones»(35). En definitiva, el 
diagnóstico realizado en vida del enfermo será ratificado con la lesión anatómica que se encuentre en la autopsia del cadáver. Por ejemplo, en el caso de un "hidro-neumo-tórax», se describen así sus lesiones: "Los síntomas expuestos en el estado actual demuestra claramente la existencia de un gran foco o caverna, inmediato a la pared torácica de la base del lado derecho, cuyos productos muy abundantes son espelidos por la tos. Los antecedentes prueban que este foco cavernoso tuvo su origen por una pleuro-neumonía en el punto afecto y de aquí puede deducirse que el derrame pleurítico primitivo se abrió paso a través del pulmón, dejando en el sitio que ocupaba una extensa caverna en supuración que bien merece el nombre de neumo-hidro-tórax por cuanto en la cavidad de la pleura se hallan acumulados un líquido sero-purulento y en comunicación con el aire atmosférico que penetra por las vías respiratorias»(36). El curso, duración, pronóstico y etiología dependen de la enfermedad concreta, si bien en las enfermedades de tipo palúdico la etiología y patogenia vienen determinadas por la permanencia del enfermo en zonas en donde se cultiva el arroz, como ya vimos antes.

Para el tratamiento, en la mayoría de los casos no se utilizan principios activos y va a ser de tipo sintomático. Se emplean balsámicos, astringentes, tónicos "neurasténicos» y analépticos. Una excepción es el tratamiento del paludismo, en el que se recurre a la quinina: «Se administró, pues - dice una historia clínica-, el sulfato de quinina 30 centígramos en 5 píldoras para tomar 3 por la mañana y 2 por la tarde...». Un detalle interesante es que se utilice la técnica china de la moxibustión. Inspeccionando el tórax se observan «las heridas de cuatro moxas que en la actualidad supuran en el lado derecho del tórax hacía el vértice del pulmón»(37). La moxibustión, que aparece utilizada en una «tuberculosis pulmonar con neuralgia cérvico-occipital» es una técnica introducida durante el siglo XIX al igual que la acupuntura.

La «Parte Complementaria» de la historia clínica consta de un «Diario Clínico», unas "reflexiones» y de una "Autopsia», si ha fallecido el enfermo. Esta última, como antes hemos dicho, es realizada en la totalidad de los fallecimientos. El diario clínico es una lista de medicamentos, regímenes alimentarios, etc. utilizados durante la estancia del enfermo en el Hospital, especificando lo que se realiza cada día.

Lo más importante de esta parte es la autopsia, reflejo de la mentalidad anatomoclínica de Peset y Vidal. Sin embargo, normalmente se complementa con la indagación microscópica, como ya hemos dicho en el apartado anterior: «El microscopio nos reveló también el desarrollo extraordinario del tejido conjuntivo del hígado, que constituye la cirrosis»(38), se 
dice en un caso de «ascitis sintomática»; «el hígado se presentó atrofiado y decolorado; desigual al tacto; cubierto de escrecencias pálidas al esterior y amarillentas en su masa, y con desarrollo estraordinario del tejido conjuntivo de la víscera que parecía hallarse atacada por un principio de esclerosis» (39). El protocolo de una autopsia correspondiente a un "encefaloides de riñón» merece ser transcrito:

Autopsia. Abierta la cavidad abdominal y separados los tegumentos se descubrió detrás del colon descendente, por fuera del paquete intestinal y al nivel del riñón izquierdo, un tumor cubierto por peritoneo al que surcaban en todas direcciones muchos vasos sanguíneos de bastante calibre e ingurgitados de sangre los venosos. Este tumor medía unos trece centímetros de diámetro vertical, diez en el transversal y ocho en el antero-posterior; su consistencia era blanda y algo pastosa en algunos puntos; su superficie después de quitada la cubierta peritöneal, se ofrecía irregular, abollonada, con eminencias semiesféricas del tamaño de una nuez, lustrosas y de color rosado pálido, que disminuía hacia la desembocadura de la arteria renal y del uréter en su borde interno. Separado el tumor que reemplazaba al riñón izquierdo, y cortado a lo largo de su borde esterno, se descubrió su parenquima de un color rosa.blanquecino y de una consistencia semipulposa. Las tres pelvis renales estaban llenas de materias purulentas en diferentes grado de consistencia, pues la superior contenía un pus claro que parecía estar mezclado con orina; sus paredes alcanzaban un espesor de ocho milímetros y su cavidad podía alojar una nuez gorda. La pelvis media contenía un pus loable, sus paredes eran gruesas de tres centímetros y su cavidad la de un huevo pequeño. La pelvis inferior capaz de contener un huevo grande y cuyas paredes tendrían tres centímetros de grueso, contenía una sustancia que parecía formada de concreciones purulentas de consistencia pastosa y tamaño variable.

El estudio microscópico del tumor reveló su naturaleza cancerosa y su forma encefaloidea (40).

En una autopsia de un corazón con hipertrofia excéntrica se llega incluso a tomar medidas del corazón, a los vasos aórticos y pesarlos: «El corazón, órgano enfermo, estaba hipertrofiado notablemente, pues pudieron apreciarse en él las siguientes dimensiones.

Longitud desde el vértice a la

parte media de la base

17 centímetros

Latitud en el punto más ancho 15 centímetros

Grosor 6 centímetros 
Grueso de las paredes en la base sin comprender las columnas carnosas 23 centímetros Grueso de las paredes en la parte media sin comprender las columnas carnosas ............ 23 milímetros

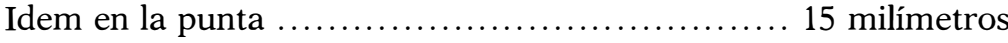
Grosor del tabique ............................. 15 milímetros Diámetro vertical desde el vértice hasta el rodete fibroso que da la inserción superior a la válvula bicúspide 15 centímetros

Cavidad de la aorta aneurismática. En su origen 8 centímetros de diámetro transversal; en el cayado 65 milímetros. La distancia desde el origen de la aorta hasta el crecimiento del tronco braquio-cefálico 10 centímetros... Peso del corazón en el tronco aórtico y el de la arteria pulmonar, 820 gramos...»(41)

Puede decirse, en suma, que en estas historias clínicas predominan los conceptos anatomoclínicos sobre el resto de las mentalidades médicas "de laboratorio», que aparecen también sin embargo, aunque de modo incipiente.

El predominio de la mentalidad anatomoclínica se ve claramente en el siguiente texto, correspondiente a una autopsia: «Estos son los datos más notables que nos suministró la importante autopsia del cadáver... La pila, pues llamada con mucha propiedad piedra de toque de la verdad o falsedad del diagnóstico, nos obliga a rectificar en parte el que hicimos en vidaw(42).

\section{NOTAS}

(1) Campos Fillol, R., (1955), Crónica de la Facultad de Medicina de Valencia (de 1866 a 1946), Ochenta años de la vida de una facultad. Apuntes para la Historia de la Medicina de Valencia, Valencia, Real Academia de Medicina; Peset Reig, J. L., (1968-1970), "La enseñanza de la medicina en España durante el siglo xıx», Medicina Española, 59, 148-157 y 381-392; 60, 28-35 y 98-105; 63, 115-130; López PIÑERo, J. M., (1980), La Facultad de Medicina de la Universidad de Valencia. Aproximación a su historia, Valencia, Universidad de Valencia; Peset ReIG, M., Peset ReIG, J. L., (1974), La Universidad española (siglos XVIII y XIX). Despotismo ilustrado y revolución liberal, Taurus, Madrid; Teruel, S. (1967), Medio siglo de medicina a través de la labor del Instituto Médico Valenciano, Tesis Doctoral, Valencia; BALDo y LACOMBA, M., (1986), La Universitat de Valencia, IVEI, Valencia. López PIÑERo, J. M., (1973), "The Development of the Basic Sciences and their influence on Clinical Medicine in 19th Century .Spain", Clio Medica, 8, 53-63; López Terrada, M. L., Pardo Tomás, J., Salavert Fabiani, V., (1988), «El marco 
institucional», en: López Piñero, J. M., et col., Las ciencias médicas básicas en la Valencia del siglo $x I X$, IVEI, Valencia.

(2) Teruel, S. (1974), La medicina en Valencia (1841-1872), CSIC, Madrid; Livianos Aløana, L. (1985), Teoría y práctica psiquiátrica en la Valencia de mediados del siglo XIX, 2 vols., Valencia, Tesis Doctoral; Peset LloRCA, V., (1987), Estudios históricos sobre la psiquiatría Valenciana, prólogo de J. M. López Piñero y J. A. Mico, IVEI, Valencia; LóPez Piñero, J. M. (1961), «Juan Bautista Peset y Vidal y las generaciones intermedias del siglo xIX médico españoly, Medicina Española, 46, 186-202; 321-322; Chiarri Llobregat, P., Necroapología del Dr. D. Juan Bta. Peset y Vidal, Valencia, Impr. de M. Alufre.

(3) Peset y Vidal, J. B., (1873), Historias clinicas. Colección estractada de las Historias Clínicas, Médicas y Quirúrgicas impresas para uso de los alumnos clínicos de la facultad de medicina de esta Universidad Literaria, Curso 1872 a 1873. Valencia. Clínica médica a cargo del Doctor D. Juan Bautista Peset profesor de dicha asignatura en esta Universidad. Curso 1872 a 1873, p. 5. En adelante citaré Peset y VIdaL, J. B., (1873), Clínica Médica... Valencia.

(4) Peset y Vidal, J. B., (1873), Clínica Médica..., p. 99.

(5) Peset y Vidal, J. B., (1873), Clínica Médica..., p. 100.

(6) Peset y Vidal, J. B., (1873), Clínica Médica..., p. 100.

(7) Peset y Vidal, J. B., (1873), Clínica Médica..., p. 101.

(8) Peset y Vidal, J. B., (1873), Clinica Médica..., p. 101.

(9) Peset y VIDAL, J. B., (1874), Historias de las Clinicas Médicas y Quirúrgicas impresas para uso de los alumnos de los mismos en la escuela de Valencia. Valencia, p. 91. En adelante citaré Peset y Vidal, J. B., (1874) Clínica Médica..., Valencia.

(10) Laín Entralgo, P., (1961), La Historia Clínica..., p. 260-261.

(11) López Piñero, J. M., (1961), «Juan Bautista Peset y Vidal y las generaciones intermedias del siglo xix médico español», Medicina Española, 46, p. 194.

(12) Peset y Vidal, J. B., (1873), Clínica Médica..., p. 22.

(13) Laí Entralgo, P., (1961), La Historia Clínica..., p. 260

(14) Peset y Vidal, J. B., (1873), Clínica Médica..., p. 77.

(15) Peset y Vidal, J. B., (1873), Clínica Médica..., p. 77.

(16) Peset y Vidal, J. B., (1873), Clínica Médica..., p. 33.

(17) Peset y Vidal, J. B., (1873), Clínica Médica..., p. 33

(18) Peset y Vidal, J. B., (1873), Clínica Médica..., p. 36.

(19) Peset y Vidal, J. B., (1873), Clínica Médica..., p. 75.

(20) Peset y Vidal, J. B., (1873), Clinica Médica..., p. 12.

(21) Peset y Vidal, J. B., (1873), Clínica Médica..., p. 55.

(22) Laín Entralgo, P., (1961), La Historia Clínica.., p. 327.

(23) Peset y Vidal, J. B., (1873), Clínica Médica..., p. 10.

(24) Peset y Vidal, J. B., (1873), Clínica Médica..., p. 29.

(25) Peset y Vidal, J. B., (1874), Clínica Médica..., p. 28.

(26) Peset y Vidal, J. B., (1874), Clínica Médica..., p. 46.

(27) Peset y Vidal, J. B., (1874), Clínica Médica..., p. 124.

(28) Laín Entralgo, P., (1961), La Historia Clínica..., p. 385, 387.

(29) Peset y Vidal, J. B., (1873), Clínica Médica..., p. 46.

(30) Peset y VIdal, J. B., (1874), Clínica Médica..., p. 140.

(31) López Piñero, J. M., y col., (1988), Las ciencias médicas básicas en la Valencia del siglo $x I X$, Valencia, p. 114.

(32) López Pinero, J. M., y col., (1988), Las ciencias médicas..., p. 21. 
(33) López Piñero, J. M. (1961), «Juan Bautista Peset y Vidal y las generaciones intermedias del siglo xIx médico español», Medicina Española, 46, pp. 186-202; 321-321.

(34) López Piñeroverx, J. M., (1961), «Juan Bautista Peset y Vidal y las generaciones intermedias del siglo xIx médico español», Medicina Española, 46. p. 194.

(35) Peset y Vidal, J. B., (1874), Clínica Médica..., p. 54.

(36) Peset y Vidal, J. B., (1873), Clínica Médica..., p. 51-52.

(37) Peset y Vidal, J. B., (1873), Clinica Médica...; p. 78

(38) Peset y Vidal, J. B., (1873), Clínica Médica..., p. 35.

(39) Peset y Vidal, J. B., (1873), Clínica Médica..., p. 42.

(40) Peset y Vidal, J. B., (1873), Clínica Médica..., p. 76.

(41) Peset y Vidal, J. B., (1874), Clínica Médica..., p. 106.

(42) Peset y Vidal, J. B., (1874), Clínica Médica..., p. 130-131. 